\title{
O \\ A
}

AIAA 2002-3140

Probabilistic Methods for

Uncertainty Propagation

Applied to Aircraft Design

Lawrence L. Green

NASA Langley Research Center

Hampton VA

Hong-Zong Lin

PredictionProbe, Inc.

Newport Beach, CA

Mohammad R. Khalessi

PredictionProbe, Inc.

Newport Beach, CA

\section{0th AIAA Applied Aerodynamics Conference}

24-26 June 2002

St. Louis, Missouri

For permission to copy or to republish, contact the copyright owner named on the first page.

For AIAA-held copyright, write to AIAA Permissions Department,

1801 Alexander Bell Drive, Suite 500, Reston, VA, 20191-4344. 


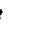

$\cdot$

.

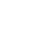

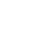

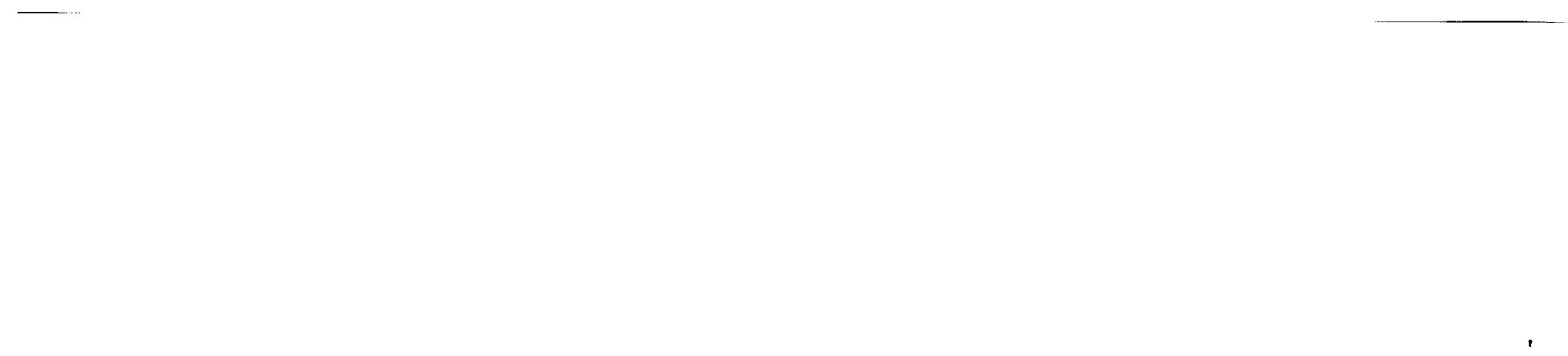
-

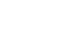

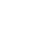


AIAA 2002-3140

\title{
PROBABILISTIC METHODS FOR UNCERTAINTY PROPAGATION APPLIED TO AIRCRAFT DESIGN
}

\author{
Lawrence L. Green ${ }^{*}$ \\ NASA Langley Research Center \\ Hampton. VA 23681-2199 \\ Hong-Zong Lin ${ }^{* *}$ and Mohammad R. Khalessi ${ }^{* * *}$ \\ PredictionProbe. Inc. \\ Newport Beach, CA 92660
}

\begin{abstract}
Three methods of probabilistic uncertainty propagation and quantification (the method of moments, Monte Carlo simulation, and a nongradient simulation search method) are applied to an aircraft analysis and conceptual design program to demonstrate design under uncertainty. The chosen example problems appear to have discontinuous design spaces and thus these examples pose difficulties for many popular methods of uncertainty propagation and quantification. However, specific implementation features of the first and third methods chosen for usc in this study enable successful propagation of small uncertainties through the program. Input uncertainties in two configuration design variables are considered. Uncertainties in aircraft weight are computed. The effects of specifying required levels of constraint satisfaction with specified levels of input uncertainty are also demonstrated. The results show, as expected, that the designs under uncertainty are typically heavier and more conservative than those in which no input uncertainties exist.
\end{abstract}

\section{Introduction}

The aerospace vehicle design process is inherently a multidisciplinary design optimization (MDO) problem ${ }^{1-42}$. Within recent years, such MDO problems have received a growing amount of attention from both the engineering and optimization communities using both gradientbased and nongradient optimization methods. Indeed, a quick survey of recent conference proceedings and of internet sources reveals

\footnotetext{
* Research Scientist. Multidisciplinary Optimization Branch. MS 159. Senior Member AIAA

"Chicl Technology Officer. 3931 MacArthur Blvd., Suite 202. Newport Beach, CA

Chief Product Development Officer. 3931 MacArthur Blvd., Suite 202. Newport Beach. CA

This material is declared a work of the U.S. Government and is not subject to copyright protection in the United States.
}

literally hundreds of papers addressing various aspects of just gradient-based optimization for aerospace configurations and its components

A mission analysis module or discipline is usually at the core of an aerospace vehicle optimization problem. For example, the major contributing disciplines of an aircraft design problem (most notably, aerodynamics, structures, and performance) may be interacted t. $^{2} 8.12 .18 .22 .28$. $41-42$ hy using an aircraft mission analysis module. Also, constraints formulated within a mission analysis module may be used to account for other contributing disciplines (such as aircraft layout) that are difficult to implement, and for features of the vehicle (such as empennage) or the mission (such as takeoff and landing) that are not the primary focus of the particular design study ${ }^{41-42}$. A particular mission analysis implementation known as the Flight Optimization System (FLOPS) for aircraft ${ }^{+3}$ is chosen here as the hasis for further study.

In many published optimization studies, the inputs to the disciplinary analyses and to the multidisciplinary optimization are assumed to be precisely known for a given problem: these studies are henceforth referred to as deterministic analyses and optimizations. In the last few years interest has grown, particularly within the structures discipline, in solving problems for which the inputs are uncertain ${ }^{4-78}$ : however. such nondeterministic optimizations are relatively uncommon in the aerodynamics and related disciplines.

Uncertainties are a prominent aspect early in the design process of a new aerospace vehicle. and these uncertainties should be accounted for in a formal way. The uncertainty in inputs for nondeterministic studies may be due to accepted approximations, unmodeled physics, a lack of knowledge ${ }^{79,80}$ about some aspect of the problem. or errors, such as a lack of precision or repeatability in measurement. or blunders 
attributed to the user or their processes. In these cases, the uncertain inputs may be considered random variables that take on some prescribed distribution of values rather than a single precise value. The uncertain input valuc distribution may be one of numerous popular distributions categorized by various statistical and probabilistic resources ${ }^{80,81^{\circ}}$. or it may be some uncategorized, perhaps even unknown. distribution that can only be approximated. These studies, in which input variables are assumed random and are drawn from a prescribed distribution of values, are henceforth referred to as nondeterministic analyses and optimizations. The potential for input uncertainty, expressed to disciplinary analyses via distributions rather than single values. introduces a level of uncertainty in the resulting analysis outputs and raises the need to consider output distributions.

A wide variety of prohabilistic uncertainty propagation techniques exist ${ }^{82-85}$. The uncertainty propagation techniques generally fall into one of six categories: simulation methods, importance sampling techniques. first-order reliability methods. second-order reliability methods. response surface methods, and method of moments techniques. This paper considers the method of moments. a Monte Carlo simulation technique, and a nongradient simulation search method. The Monte Carlo simulation technique is only used for comparison with the method of moments approximation.

The application of probabilistic methods requires the definition of (1) one or more random input variable probability models or distribution types. (2) one or more response models that describe the physics, process, or rules which govern the system hehavior, and (3) one or more models that predict the outcome of an event; these predictive models are generally called limit states. The intent of each of these uncertainty propagation techniques is to evaluate a multidimensional probability integral over a multidimensional surface known as the limit state. However, in practice, evaluating this multidimensional integral in closed form is problematic for several reasons: (1) the joint pdf is generally not known. (2) the boundary over which the integral is to be evaluated (the limit state) is generally not known. and (3) even when the pdf and limit state are known, the multidimensional integral itself is difficult to evaluate. As a result. various uncertainty methods, each with differing computational features, and levels of accuracy and efficiency. have been proposed and developed to circumvent these difficulties.

The random variable probability models for this study are chosen for convenience from among many possible distribution types; for example, the variable probability models could be described by normal, lognormal. Weibull, uniform. or beta distributions. For each distribution type, the pdf describes the probability that a certain value of the random variable will occur. plotted as a function of the range of possible values that can be assigned to the random variable. The shape of these distributions is generally described analytically by at least two parameters, including the mean value (denoted herein by variables with an overbar) and the standard deviation $(\sigma)$, which is a measure of the dispersion of the random values about the mean value. Some distributions may require more than two parameters to be described, but the mean value and standard deviation are sufficient for the distributions considered herein. The standard deviation is the product of a random variable mean value and the more commonly chosen coefficient of variation (c.o.v.). The reader should note that the normal, lognormal, and Weibull distributions are unbounded in at least one direction, whereas the uniform and beta distributions are bounded in both directions. In this paper, only normal distributions are used for input variables.

Each pdf has an associated cumulative distribution function (cdf)describing the probability that the value of a random variable is less than or equal to some prescribed value taken from the total range of possible values. Two or more random variables may be correlated (dependent, or unrelated but changing together), or uncorrelated (totally independent); in this paper. only truly independent random variables with normal distributions are considered.

A given uncertainty analysis might yield one or more of the following results: identification of a single most probable point (mpp), or a locus of mpp, at which a certain event might occur; computation of the reliability index and its gradients with respect to the mean values and standard deviations of the random input variables at the MPP: computation of the parameters describing a random output pdf or cdf given one or more known random input pdf and cdf; approximation of the output pdf or cdf shape without the formal calculation of the parameters associated with such a distribution: or the accommodation of uncertainty in random input variables without approximating the pdf and edi. The method of moments used in this paper accommodates uncertainty in random input variables and produces estimates for the mean value and the standard deviation of the output variables. These estimates are accurate only if the resulting output distribution is normal. The Monte 
Carlo simulation method can approximate the pdf of an output function for any input distribution, without actually computing the parameters needed to describe the pdf shape, but requires many function evaluations for accuracy. In contrast, the nongradient simulation search method (ssm) can efficiently produce very accurate representations of the pdf and edf for any distribution types, as well as identifying the mpp. the reliability index, and the gradients of the reliability index with respect to the random input variable mean value and standard deviations at the mpp.

An uncertainty propagation and quantification demonstration for a onedimensional inviscid acrodynamic example problem, using normal distributions for two input random variables, was developed in a recent paper by Putko et al. ${ }^{86}$. Parameters for the output distributions were computed by the method of moments and verified by Monte Carlo simulations. Other work in aerodynamic uncertainty propagation and quantification has since been published involving the extension to twodimensional airfoil design under uncertainty ${ }^{87.88}$ and the propagation of uncertainty through aerodynamic viscous and turbulent flows via the application of random field theory ${ }^{89,90}$. The work of Putko ${ }^{86}$ was also the basis for similar uncertainty propagation work in a threedimensional aerodynamic/structural interaction by Gumbert et al. ${ }^{91}$. The Putko and Gumbert work closely followed the work of others from the structures discipline. most notably ${ }^{92-93}$. which showed that a statistical first-order second-moment (FOSM) method and automatic differentiation (AD) could be used to efficiently propagate the input uncertainties through finite element analyses to approximate the output uncertainty. An integrated strategy for mitigating the effect of uncertainty in simulation-based design is presented $\mathrm{in}^{13}$; il consists of uncertainty quantification. uncertainty propagation. and robust design tasks or modules. Two approaches are developed in ${ }^{93}$ to propagate uncertainty through sequential analysis codes: an extreme condition approach and a statistical approach. The latter approach can be efficiently implemented using the FOSM approximation and sensitivity derivatives (SD) as was done by Putko.

The response model for this demonstration is the FLOPS mission analysis code. which includes equations to compute the aircraft weight, range, cost, noise metrics, and other readily available performance constraints. The method of moments was implemented in the FLOPS aircraft mission analysis program for several classes of statistically independent, normally-distributed, random input variables and several classes of random output variables, noted above. However, due to concerns about the accuracy and applicability of the method of moments for this code, only uncertainty propagation results for examples with two uncertain input variables and one uncertain output variable are shown in this paper. Different levels of input uncertainty and required constraint satisfaction are imposed. The effect of uncertainty on the design point, compared with a deterministic design. is noted. Output distributions from the deterministic code are compared with Monte Carlo simulations. Sample results from the ssm technique of the UNIPASS ${ }^{\text {TM }}$ tool are also shown.

The problem (including the FLOPS mission analysis code, the aircraft, its mission, design variables, objective, and constraints) was chosen for this uncertainty demonstration because this particular mission analysis code executes quickly, which enables some level of validation with Monte Carlo techniques. Furthermore, the FLOPS code has been shown to be very amenable to processing ${ }^{42}$ by the Adifor automatic differentiation tool ${ }^{100-105}$, which enables very efficient computation for thousands of derivatives. Unfortunately, the FLOPS code also produces a large number of failed analyses (illustrated subsequently) when executed for a series of related cases, as might be done during Monte Carlo simulation. The frequent failures of the analysis code result in a design space that. to an optimization or uncertainty propagation tool, appears to be discontinuous. This situation is actually a common feature of many complex analysis codes. It is also a feature that is not wellhandled by many commercially developed tools. But the discontinuous nature of the FLOPS design space serves as a good example to illustrate the benefits of a nongradient technique available in the UNIPASS $^{\mathrm{TM}}$ tool. In this paper, all work related to and using the UNIPASS ${ }^{\mathrm{TN}}$ tool was performed by the PredictionProbe, Inc. experts, whereas the method of moments work was performed by the NASA civil servant.

\footnotetext{
"The use of trademarks or names of manufacturers in this report is for accurate reporting and does not constitute an official endorsement, either expressed or implied, of such products or manufacturers by the National Aeronautics and Space Administration.
} 


\section{Approach and Methods}

\section{The FLOPS Mission Analvsis Code}

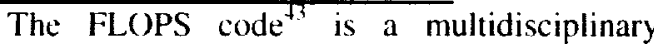
system of computer programs for conceptual and preliminary design and evaluation of advanced aircraft concepts. It consists of nine primary modules: weights. aerodynamics, engine cycle analysis. propulsion data scaling and interpolation. mission performance, takeoff and landing, noise footprint, cost analysis. and program control.

The FLOPS code may be used to analyze a point design, to parametrically vary certain design variables, or to optimize a configuration with respect to numerous design variables using nonlinear programming techniques. A variety of configuration. mission performance, noise abatement, and engine design variables and a composite objective function (including the minimum gross weight, minimum fuel burned, maximum range, minimum cosl. and/or minimum NO, emissions) are provided to allow for simultaneous optimization of the aircraft configuration. engine cycle, and size.

Two example cases. distributed with the FLOPS code, will be used for the uncertainty propagation demonstrations in this paper. The first is a subsonic aircraft transport design case. The second is a supersonic aircraft transport design case.

\section{The ADIFOR Automatic Differentiation Tool}

The ADIFOR ${ }^{1 / 2-105}$ sofiware package is a

tool for the AD of standard FORTRAN 77 programs. Given a FORTRAN 77 source code and user-specified dependent and independent variables. Adifor will formulate exact derivatives (via repeated and systematic application of the chain rule of calculus) and generate new FORTRAN 77 code. The new code includes original function evaluation, augmented with code that computes the partial derivatives (gradient) of the specified dependent variables with respect to the specified independent variables.

The ADIFOR 2.0 software package ${ }^{102}$ provides a production-quality $\mathrm{AD}$ environment that can compute derivatives by the forward (direct) mode of AD. In the forward mode of AD. the gradient code execution time and memory are usually proportional to the number of independent variables; this technology is best suited to problems in which the number of dependent variables is greater than the number of independent variables.

The ADIFOR 3.0 software package $\mathrm{e}^{103-165}$ includes both forward and reverse (adjoint) modes of $\mathrm{AD}$ for first derivatives, and three forward mode options for computing second derivatives. In the reverse mode of $A D$, the gradient code execution time and memory are usually proportional to the number of dependent variables: adjoint technology is best suited to problems in which the number of independent variables is greater than the number of dependent variables. Previous studies with the FLOPS code $e^{42}$ indicate that this program requires only a few minor changes to correct nonstandard FORTRAN 77 coding to enable ADIFOR 2.0 processing of the code to compute first derivatives. The resulting gradient code was also found to be exceptionally efficient in computing thousands of first derivatives via the forward mode of differentiation; it was almost as quick as the original code execution. Both ADIFOR 2.0 and ADIFOR 3.0 were applied to the FLOPS code during the course of this study: in fact. several "hugs" in the ADIFOR 3.0 package and in the FLOPS code were identified and corrected as a result of this work. However, only results using ADIFOR 2.0 are shown in this paper.

\section{ADIFOR Application to FLOPS}

The independent variables for differentiation were selected from among the possible configuration, mission performance, and noise abatement design variables input to FLOPS. The independent variables for differentiation also included representative elements in the aerodynamic and propulsion data provided to the code from external sources. The dependent variables were selected from numerous outputs from the FLOPS analysis, including the composite design objective, the takeoff gross weight. the vehicle life cycle cost per unit, a noise metric, an emissions metric, and seven typical constraints from a menu of nineteen that could be activated for the problem.

Since the FLOPS analysis is embedded within the optimization, extra care was taken to ensure that correct derivatives were obtained for the analysis portion of the code during both analysis and optimization modes of the code execution. This entails allowing for differentiation with respect to the input design variable values (used for analysis mode, and as the starting point for optimization) and with respect to the local design variable values used in the analysis module when embedded within the optimization mode. It also required special handling of the derivative activation ("buddy variable") sites within the code, the derivative seeding to an identity matrix, and the zeroing of certain iterated variables to ensure that derivative objects were not contaminated 
during analysis $\mathrm{N}$ with information from analysis $\mathrm{N}-1$. It is expected that any analysis embedded within an optimization will require similar techniques to ensure proper derivative evaluation.

The computed derivatives were only used to provide gradients needed for the uncertainty augmentation. described subsequently. ADIFOR could also be used to provide the derivatives needed for the gradient-based optimization within FLOPS; however, that was not done here. Higher order derivatives could also be obtained via ADIFOR differentiation to enable the formulation of higher order uncertainty corrections (more accuracy) and higher moment approximations (more information about the output distributions).

\section{The UNIPASS ${ }^{\text {TM }}$ Software}

The UNIPASS ${ }^{\text {TM }}$ software, developed by PredictionProbe, Inc.. is a general-purpose Unified Probabilistic Assessment Software System that performs complex probabilistic analyses. The UNIPASS $^{\mathrm{TM}}$ software can be utilized independently as a stand-alone software engine, and/or integrated with deterministic software tools. UNIPASS $^{T M}$ provides the basis for modeling uncertainties, constructing probabilistic predictive models. computing probabilities, identifying most likely outcomes, providing sensitivity data. identifying key drivers, analyzing risk. and performing sensitivity analysis; deterministic software tools may be integrated to provide the computational framework for constructing complex deterministic process models. The latest version. UNIPASS ${ }^{\mathrm{TM}} 4.2$, offers an advanced graphical Windows environment, 2-D and 3-D graphic functions, four problem types (component. serial. parallel, and general), three analysis types (probability, inverse probability, and cdf/pdf analysis), six categories of prohabilistic methods, thirty-seven probability distribution types that can be used to define any type of random variahle, four classes of random variables, three ways to interface with any commercial and/or in-house software tools. eleven gradient-based mpp identification methods. and one nongradient simulation-based search method (SSM) that finds the mpp for discontinuous, nondifferentiable limit state functions. In general, the mpp represents the most likely values of the random variables at which the critical or significant condition of the user-defined event will occur. In engineering. a critical condition may be an undesirable event such as component failure or instability, or a desirable event such as extended component life or mission success. Some software products use only a gradient-based algorithm to identify an mpp. However. those algorithms are limited to continuous and differentiable variables, and cannot handle the more common engineering tasks, such as the FLOPS examples studied herein. which involve discontinuous limit state functions.

\section{Uncertainty Propagation}

\section{Method of Moments}

Uncertainty propagation is accomplished by using various orders of approximations to the various statistical moments: this is a logical naming convention for the uncertainty propagation technique results from a given choice of the order of approximation and the statistical moment to be used. Only the FOSM approximation is used for results in this paper.

In this study, the effects of uncertainty in two input aircraft design variables are considered for the purposes of illustrating uncertainty propagation through the FLOPS code and design under uncertainty. For the present demonstration (and following the derivation in ${ }^{88}$ and 89 ). these input variables are assumed statistically independent, random, and normally distributed about a mean value. These assumptions simplify the implementation and help quantify the input uncertainties. The assumption of the variables being statistically independent is not required: correlation between the variables can be easily accounted for within the formulation at the cost of more computational work. For non-normal input distributions, the method of moments corrections are only approximately correct. In this case, the increase in accuracy, gained from considering additional terms in the Taylor series expansion. could very well be offset by the approximation error due to the non-normal nature of the input variable.

Given a vector $B=\left\{b_{1}, \ldots, b_{n}\right\}$ with $n$ independent input random variables, $b_{i}$ (for $i$ $=(, n)$, mean values $\bar{B}=\left\{\bar{b}_{1}, \ldots, \bar{b}_{n}\right\}$, standard deviations $\sigma_{b}=\left\{\sigma_{b_{1}}, \ldots, \sigma_{h_{n}}\right\}$, and random output function $F$, first-order $(\mathrm{F}())$ and second-order (SO) Taylor series approximations to the function are given in generic form by

$\mathrm{FO}$ :

$$
F(B)=F(\bar{B})+\sum_{i=1}^{n} \frac{\partial F}{\partial b_{i}}\left(b_{i}-\bar{b}_{i}\right)
$$


SO:

$$
\begin{aligned}
& F(B)=F(\bar{B})+\sum_{i=1}^{n} \frac{\partial F}{\partial b_{i}}\left(b_{i}-\bar{b}_{i}\right)+ \\
& \frac{1}{2 !} \sum_{i=1}^{n} \sum_{i=1}^{n} \frac{\partial^{2} F}{\partial b_{i} \partial b_{i}}\left(b_{i}-\bar{b}_{i}\right)\left(b_{i}-\bar{b}_{i}\right)
\end{aligned}
$$

where the first and second derivatives are evaluated at the mean values. $\bar{b}_{i}$ and $\bar{B}$.

One must then obtain the expected values for the mean (first-moment) and variance (secondmoment) of the output function, $F$, which depend on the derivatives of $F$ with respect to the uncertain input variables and input variances, $\sigma_{b}{ }^{2}$. The expected values of a random function are obtained from the integration of the product of the function itself and the imposed pdf. For normally distributed input values. the pdf is symmetric about the mean value. Thus, the expected value of an odd function with a normal input distribution involves the integration of the product of an even and odd function, which is zero. Likewise, the expected value of an even function involves the integration of the product of two even functions, which is nonzero. The mean value of the output function $\bar{F}$ and standard deviation $\sigma_{F}$, are approximated (as in ${ }^{86}$ ) as

$\mathrm{FO}$ :

$$
\begin{aligned}
& \bar{F}=F(\bar{B}) \\
& \sigma_{F}^{2}=\sum_{i=1}^{n}\left(\frac{\partial F}{\partial b} \sigma_{i}\right)^{2}
\end{aligned}
$$

\section{SO:}

$$
\begin{aligned}
& \bar{F}=F(\bar{B})+\frac{1}{2 !} \sum_{i=1}^{n} \frac{\partial^{2} F}{\partial b_{i}^{2}} \sigma_{i}^{2} \\
& \sigma_{i}^{2}=\sum_{i=1}^{n}\left(\frac{\partial F}{\partial b_{i}} \sigma_{l_{i}}\right)^{2}+\frac{1}{2 !} \sum_{j=1}^{n} \sum_{i=1}^{n}\left(\frac{\partial^{2} F}{\partial b_{i} \partial b_{i}} \sigma_{l,} \sigma_{b_{i}}\right)^{2}
\end{aligned}
$$

where the first and second derivatives are again evaluated at the mean values. $\bar{b}_{i}$ and $\bar{B}$. Note in Eq. (4) that the second-order mean output $\bar{F}$ is not at the mean value of input $\bar{b}$; a shift in the mean value of the uncertain output function occurs due to the specified input uncertainty, i.e., $\bar{F} \neq F(\bar{B})$.

\section{Deterministic Optimization}

For simplicity, a demonstration of deterministic optimization is derived from two particular sample cases distributed with the FLOPS code. The first example uses the inputs for the FLOPS five-design variable subsonic transport design (xfp2.in). The input file is modified to allow only the variables THRUST (the maximum rated thrust per engine, in pounds force), and SW (the wing reference area, in square feet), to be active design variables; upper and lower bounds have also been specified for these design variables. The optimization objective is specified to be the aircraft gross takeoff weight. The seven possible aircraft performance constraints. normally activated with this sample problem. are used. These include the aircraft required range (which is held fixed for this problem), the approach speed, the takeoff and landing distances, the approximate missed-approach and second-segment climb gradients, and the excess fuel. The BroydenFletcher-Goldfarb-Shano (BFGS) optimization method (the default among several optimization methods available within the FLOPS code) was used to solve this problem. In the FLOPS implementation of this optimization method, a composite objective function is minimized. The composite objective function is composed of the true objective augmented with a highly nonlinear penalty function that grows rapidly as the design variables approach their upper or lower bounds, and as constraints become active.

\section{Robust Optimization}

The form of the uncertain objective follows from the development in ${ }^{86}$, with an adaptation to the current optimization problem. The FOSM expression for the current uncertain objective is

$$
O b j=\sqrt{\overline{O b j^{2}}\left(1.0+\frac{\sigma_{O l_{j}}^{2}}{O b j^{2}}\right)}
$$

where

$$
\sigma_{i t_{i}}^{2}=\sum_{i=1}^{n}\left(\frac{\partial O b j}{\partial b_{i}} \sigma_{b i}\right)^{2}
$$

and where the $\sigma_{b}$ are the known standard deviations for each of the random input variables. as noted previously. The adaptation of the uncertain objective provides a composite objective function, similar in magnitude and functionality to the original objective function of the FLOPS code but augmented with uncertainty effects. Since the uncertainty correction to the objective function is small compared with the uncertainty correction to 
the constraints, it may be possible to neglect the objective uncertainty correction altogether; this is proposed as the subject of further research.

Again, following the development in ${ }^{86}$. the forms of uncertain constraints are written as:

$g_{m}=\overline{g_{m}}+k \sigma_{g_{m}} . \quad m=1.7$

where

$$
\sigma_{s_{m}}=\sqrt{\sum_{i=1}^{n}\left(\frac{\partial g_{m}}{\partial b_{i}} \sigma_{b_{i}}\right)^{2}}, \quad m=1,7
$$

and where $k$ is the required probahilistic constraint satisfaction in units of input variable standard deviations. For this example, values of $k$ ranging from zero (constraint satisfied with $50 \%$ probability for a normal distribution) to three (constraint satisfied with $99.9 \%$ probability for a normal distribution) were considered. Values of the random variable $\sigma_{n}$, were each computed as the product of a c.o.v. and the random input variable mean value, both input to FLOPS program, for each variable. The c.o.v. values were chosen to be the same for all the random input variables in a given problem.

It is surprising that tiny numerical differences between the deterministic and robust solutions (effects of uncertainty) could be ohserved for extremely small values of the input c.o.v. (i.e., for c.o.v. of order $\left.10^{-20}\right)$. These solutions are "deterministic" from a practical point of view, but exhibit numerical behavior that can only be attributed to the imposed uncertainty corrections. However, for "small enough" values of c.o.v. (i.e., for c.o.v. of order $10^{-30}$ ), no differences from the purely deterministic solution could be discerned, as expected.

The method of moments formulation described above requires derivatives to be evaluated. If these derivatives were to be computed by finite difference approximations, it would be necessary to find two or more successful function evaluations near the point of interest for evaluating the derivative. For the example problems chosen, this requirement was sometimes difficult to meet because of frequent failures of the analysis module, which led to discontinuous objective and limit state functions, although the design physical space of interest was locally (or piecewise) continuous. The discontinuous nature of the design space with the FLOPS code also caused problems for the UNIPASS ${ }^{1 \mathrm{M}}$ tool, necessitating the use of the nongradient SSM. However, the use of automatic differentiation to compute the derivatives made the method of moments formulation more successful than might be expected for examples with numerical discontinuities because the derivatives were evaluated analytically at each point of interest via the chain rule. Thus, finding successful neighboring points to a successful analysis point was not an issue with the current method of moments uncertainty formulation.

Two restrictions that are more serious do arise from using the method of moments: (1) the formulation is only valid for normal input and output distributions. and (2) the function gradients are evaluated at the mean value point, rather than at the mpp. Thus, the method of moments computations are generally not very accurate away from the mean value of the random function and are even less accurate for non-normal distributions. In particular, the method of moments is expected to be significantly inaccurate for predicting very low probability of failure points (e.g.. in the tail region of a normal distribution) and for highly skewed output distributions. The use of $\mathrm{AD}$ to evaluate gradients offers two possibilities to address this issue: (1) if higher order derivatives are available, better approximations can be achieved for low probability failure points by constructing Taylor series approximations that better represent those evaluation points far from the mean value, and (2) if the mpp can be identified by other means, the functional derivatives can be obtained at the mpp via $A D$ without the need to find successful points neighboring the mpp.

\section{Simulation Search Method (SSM) of UNIPASS ${ }^{\text {TM }}$}

The FLOPS optimization routines, like many other optimization algorithms, may not produce acceptable results for a given set of input data, and therefore will result in a highly discontinuous response surface. When probabilistic analysis is performed, such discontinuity in the response surface cannot be handled with standard first- and second-order reliability methods or other approximate and more efficient probabilistic techniques. Furthermore, since the desired level of reliability is usually greater than 0.99999 , the application of Monte Carlo simulation becomes impractical. Therefore, an efficient first- and second-order reliability method that could solve such nondifferentiable discontinuous problems, 
such as the one provided by UNIPASS ${ }^{\mathrm{TM}}$, becomes highly desirable.

The UNIPASS ${ }^{\mathrm{TM}}$ nongradient SSM was applied to the FLOPS code for the sample problem halsed on the subsonic transport design problem, again modified to allow only the THRUST and SW to be active design variables. Application of the SSM also allowed for: (1) determination of the aircraft weight PDF for a probability range from 0.01 to 0.99 , (2) determination of the aircraft weight CFD and the maximum aircraft weight due to input uncertainties to a probability of 0.9999 . (3) mpp identification, and (4) computation of the gradients of the reliability index.

\section{$\underline{\text { Results }}$}

\section{Method of Moments}

Starting from the subsonic transport optimization problem ("xflp2.in") test case distributed with the FLOPS code, the input file was first modified so that only THRUST (maximum rated thrust per engine) and $S W$ (the reference wing area) in Namelist \$CONFIN were active design variables. These variables are the primary aircraft sizing variables. The modified input file was then used for both deterministic and robust optimizations. The robust optimizations had various levels of input uncertainty for the active design variables and various levels of required constraint satisfaction, both specified in auxiliary input file to FLOPS. For the first example case, the specified uncertainty corresponds to a c.o.v. of $5 \%$ for each of the two input variables. Since the mean value of THRUST for this problem is 47,500 It and the mean value of $S W$ for this problem is $2272 \mathrm{ft}^{2}$. one standard deviation $(\sigma)$ is $2375 \mathrm{lb}$, and $113.6 \mathrm{ft}^{2}$ for the two variables, respectively. Figure 1 is a simplified aircraft sizing contour ("thumbprint") plot illustrating the design space near the deterministic and robust optimizations for this case. The $x$-axis of the figure shows a normalized value of the maximum rated thrust per engine, ranging from 20.000 to 60,000 pounds force: the $y$-axis of the figure shows the normalized value of the reference wing area. ranging from $1000105000 \mathrm{ft}^{2}$.

The figure is simplified from typical thumbprint plot in that only the active constraint violation boundaries for the specified problem are shown, rather than multiple contours representing various values of the limit state function. The constraint violation boundaries were interpolated from a parametric variation of the two variables with nine equally spaced points in each direction. Also shown in figure $I$ are the locations of final design points for the deterministic and three mean robust optimizations. In this example, both FLOPS constraint 2 (the upper limit on approach speed) and FLOPS constraint 5 (the lower limit on missed approach climb gradient) were active for the deterministic optimization. As expected, the deterministic design point is found at the intersection of the two active constraints. The optimization path was almost entirely within the feasible region in the figure.

The three robust optimization points (labeled $k=1, k=2$, and $k=3$ in the figure) correspond to imposed constraint satisfaction margins of 1,2 , and 3 standard deviations about the mean value of the deterministic solution. The offset in the robust design points from the constraint violation boundaries is proportional to both the imposed input uncertainty and the gradient of the constraint with respect to the uncertain design variables. Figure 1 also shows that the robust optimization with $k=1$ enforces a greater margin of satisfaction for both constraints than does the deterministic optimization. Similarly, each of the robust solutions enforees greater constraint satisfaction, with increasing values of $k$ than either the deterministic solution or the robust optimizations with smaller values of $k$. For the deterministic optimization. the constraint satisfaction with respect to single constraint violation is only $50 \%$ probability for an output normal distribution; for $k=1$, this probability increases to about $84 \%$; for $k=2$, the probability is about $97.7 \%$ : and for $k=3$, the probability rises to about $99.9 \%$.

Simultaneously (not shown in the plots), the aircraft weight increases with increased constraint satisfaction from the deterministic value of $213110.5 \mathrm{lb}$ to a value of $217052.6 \mathrm{lb}$ for $k=1$, to a value of $220222.0 \mathrm{lb}$ for $k=2$, and to a value of $223443.1 \mathrm{lb}$. for $k=3$. The weight for $k=3$ is about $5 \%$ higher than the deterministic solution.

Similar results to those shown in figure 1 are presented in figure 2 , this time for a c.o.v. of $10 \%$. The relative offset from the constraint violation boundaries grows in proportion to the increased input uncertainty imposed on this problem, relative to the previous example. Simultaneously (not shown in the plots), the aircraft weight increases with increased constraint sutisfaction. The deterministic solution has a weight of $213110.5 \mathrm{lb}$; $k=1$ has a weight of $220218.9 \mathrm{lb} ; k=2$ has a weight of $227079.4 \mathrm{lb}$; and $k=3$ has a weight of $234092.7 \mathrm{lb}$ (about $10 \%$ greater than the deterministic weight).

Generally, similar results to those shown in figures 1 and 2 are shown for the supersonic 
transport optimization problem ("xflp3.in") test case in figures 3 and 4 with violation boundaries constraints 2 and 3 (takeoff distance). In this case, the design under the uncertainty problem was much more difficult to solve in a robust design mode: the design path was almost entirely in the infeasible region of the figure, meaning the uncertainty correction contributed substantially to the nonlinear penalty function. The example exhibited much greater sensitivity to smaller levels of input uncertainty and to smaller levels of variation in local gradients calculated for objectives and constraints at various points in the design evolution. The deterministic design point appears to be caught in a corner of constraint 2 , rather than at the intersection of the two sometimes active constraints. In many cases, the optimization path was significantly different under small levels of input uncertainty than for the deterministic optimization. Figure 3 shows the results for only $0.05 \%$ variation of the THRUST and SW. The same general behavior is observed as in figures 1 and 2 . but figure 3 shows much greater levels of sensitivity to the level of input uncertainty. Figure 4 shows the results for $0.08 \%$ variation in the same two input variables.

Results from a 5000 sample Monte Carlo simulation, centered at the deterministic design point of figures 3 and 4 and based on ${ }^{106}$, are shown in figure 5. Of the 5000 requested FLOPS analyses, $447(8.94 \%)$ failed to produce an answer. Over the course of preparing this paper, FLOPS analysis failure rates ranging from $8.2 \%$ to $88 \%$ were observed during various NASA civil servant attempts to perform Monte Carlo simulations, depending on the various parameters chosen to guide the Monte Carlo simulation. The average analysis failure rate from seven Monte Carlo attempts was $28 \%$, which agrees closely with the FLOPS analysis failure rate observed by the experts from PredictionProbe during the course of their studies. The FLOPS gross weight response (labeled Prob in the figure) was converted to a standard normal space for comparison with a standard normal distribution (labeled Norm in the figure). The figure shows that the FLOPS output distribution departs significantly from that of a normal distribution. suggesting a highly nonlinear response from the FLOPS code for this supersonic transport design case. The mean value shifts significantly from that of a standard normal response and even a bimodal response pattern is observed, indicating that the lower aircraft weights could be found "just around the corner" from the deterministic optimization point. This result might be expected after examining the location of the deterministic design point in figures 3 and 4 at a corner of a single constraint in the design space.

Despite the obviously non-normal output behavior of the FLOPS code for this example, as depicted in figure 5 , on-going research suggests that the failure rates with respect to constraint violation predicted by the method of moments are similar in magnitude to those predicted by Monte Carlo simulations centered about the various design points depicted in figures 1-4. More research into the accuracy of the method of moments, perhaps utilizing $\mathrm{AD}$ to compute higher order approximations to higher moments, might be warranted in this case.

\section{UNIPASS ${ }^{\mathrm{TM}}$ Example}

A pdf/cdf analysis was performed for the subsonic aircraft design problem using the SSM to determine the aircraft weight distribution for the probability range from 0.01 to 0.99 (the middle $98 \%$ of the weight distribution). The SSM allows for identification of the mpp and calculation of the reliability and sensitivity data for nondifferentiable discontinuous problems. Assuming THRUST and SW to be normally distributed with mean values of 34405.11734 and 2054.19523, respectively. figures 6 and 7 depict the pdf and cdf of the aircraft weight for the deterministic optimization shown in figures 1 and 2, for c.o.v. $=5 \%$ and 10 $\%$, respectively as determined by the UNIPASS ${ }^{\mathrm{TM}}$ tool. Note that the output distributions are not normal distributions, which implies that the results shown in figures 1 and 2 for the method of moments uncertainty propagation may also be suspected of inaccuracy.

The SSM is much more accurate than a comparable Monte Carlo simulation, for a given number of analyses, at predicting the output distribution cdf/pdf. For example, more than 40.000 successful runs would be needed to produce an accurate edf point for a probability level of 0.99 with c.o.v. $=0.05 \%$. Furthermore while a 0.99 probability level may be adequate to predict the potential overweight, it is far from adequate for reliability estimates or risk calculations, which often require success probability levels of $0.99999+$. In such cases, $40,000,000+$ successful runs are needed to predict probabilities with c.o.v. $=0.05 \%$, which is impractical for real world applications.

Alternatively, an SSM approach may be used to develop the cdf/pdf of the aircraft weight significantly fewer runs. In this case, the number of the maximum number of runs is determined by the SSM based on a predefined tolerance level. Using SSM, 457 executions of FLOPS produced 
26.3 successful runs ( $42.4 \%$ failure rate), which were used to identify an mpp, to produce the cdf plots (figures 6 and 7 ), and to generate sensitivity measures (figures 8 and 9). The total number of successful runs required to produce accurate values for probability values of $0.99999+$ is not expected to increase significantly.

Sample sensitivity data from UNIPASS ${ }^{\mathrm{TM}}$ showing the sensitivity of the reliability index with respect to the mean values of the input random variable. evaluated at the mpp and scaled by the random variable mean values, are depicted in figures 8 and 9 , for c.o.v. $=5 \%$ and $10 \%$, respectively. Significantly, these figures indicate that there are three distinct regions in the design space: (1) negative sensitivities for both SW and THRUST, (2) positive sensitivity for THRUST and negative sensitivity for SW. and (3) positive sensitivities for both variables. Additional studies are needed to understand the implications and limitations of this sensitivity information for the given problem before drawing any conclusions. For example, recall that these Iwo variables were deemed to be uncorrelated (without discussion), when, in fact. greater SW would logically require greater THRUST. Subsequent studies should fully consider any possible statistical correlation between the uncertain variables. It should also be emphasized that this probabilistic study was performed using only two uncertain variables. whereas the potential number of deterministic and uncertain variables could be significantly more for many cases of interest.

\section{Conclusions}

The FLOPS aircraft mission analysis and optimization code was successfully augmented with approximations to the first-order secondmoment probahilistic uncertainty propagation terms for the objective and potentially active constraints. Two input variables that substantially contribute to aircraft shape and sizing were assumed to be uncertain in two separate test cases: a subsonic transport design and a supersonic transport design. These variables were assumed to be statistically independent and to take on random. normally distributed input values about a mean value. Gradients required for uncertainty augmentation were obtained by using automatic differentiation applied to the code.

Results from two deterministic optimizations and from several designs under uncertainty, with various amounts of imposed uncertainty for two input design variables, were presented. For the subsonic transport design case. input uncertainties of $5 \%$ and $10 \%$ of the mean value of the uncertain input variables were considered. Results were also shown for increasing amounts of required constraint satisfaction. As expected, the weight of the aircraft increases in all cases from its deterministic value. The amount of weight increase was proportional to both increasing amounts of uncertainty and to increasing amounts of required constraint satisfaction specified in the optimization problem.

For the subsonic transport design problem. the output probability density function distributions computed by a commercially distributed uncertainty propagation tool are nonnormal in shape, indicating a nonlinear response from the code for which the method of moments is known to be inaccurate. For the supersonic transport design case, uncertainties of only $0.08 \%$ of the mean value of the uncertain input variable were considered. They produced levels of output uncertainty similar to those for the subsonic transport example with 10\% input uncertainty. The weight distribution also departed significantly from that of a standard normal distribution. making the application of the method of moments highly questionable for this case. Although the theoretical accuracy of the uncertainty propagation results obtained with the method of moments is questionable for the cases shown. in practice, the method may still yield reasonable approximations for the constraint failure rate. Further research into the accuracy of the method of moments approximation is recommended for both cases.

The FLOPS code was also successfully integrated and analyzed with a commercially distributed probabilistic assessment software system to identify aircraft weight probability density function, cumulative density function. and maximum weight to a high probability level. and the most probable point (mpp) of failure with respect to the imposed design constraints. Since the analysis module under consideration exhibited significant numerically discontinuous behavior. the mpp was found using a nongradient simulation search method. Once the mpp was known, an inverse probability technique was applied to compute sensitivities of the reliability index with respect to the mean value of two random input variables. The probability density function and cumulative density function of the random output variable were also computed.

\section{References}

1. Guruswamy, G., "Coupled Finite Difference/Finite-Element Approach for 
Wing-Body Aeroelasticity." AIAA Paper 92-4680, Sept. 1992.

2. Gage, P.. and Kroo, I. "Development of the Quasi-Procedural Method for Use in Aircraft Configuration Optimization." AIAA Paper 92-4693, Sept. 1992.

3. Rais-Rohani, M.. Haftka, R., Grossman. B., and Unger. E., "Integrated Aerodynamic-Structural-Control Wing Design." AIAA Paper 92-4694, Sept. 1992.

4. Unger, E., Hutchinson, M.. Huang. X.. Mason, W.. Haftka, R., and Grossman. B., "Variable Complexity AerodynamicStructural Design of a High-Speed Civil Transport." AIAA Paper 92-4695. Sept. 1992.

5. Volk, J.. "Multidisciplinary Design Environment Development for Air Vehicle Engineering." AIAA Paper 921113, Sept. 1992.

6. Coen, P.. Barthelemy, J.-F., and Wrenn. G.. "Integration of Aerodynamics and Performance in the Preliminary Design of a Supersonic Transport," AIAA Paper 92-4718. Sept. 1992.

7. Stubbe, J., "PAYCOS: A Multidisciplinary Design Tool for Hypersonic Vehicle Design." AIAA Paper 92-4723, Sept. 1992.

8. Dovi, A., Wrenn. G., Barthelemy. J.-F.. and Coen. P., "Design Integration for a Supersonic Transport Aircraft," AIAA Paper 92-4841. Sept. 1992.

9. French, M., and Eastep. F.. "Acroelastic Model Design Using Structural Optimization." AIAA Paper 92-4730. Sept. 1992.

10. Livne, E.. "On the Optimal Size and Location of Control Surfaces in Integrated Multidisciplinary Wing Synthesis," AIAA Paper 92-47.35. Sept. 1992.

11. Olds. J., "The Suitability of Selected Multidisciplinary Design and Optimization Techniques to Conceptual Aerospace Vehicle Design." AlAA Paper 92-4791, Sept. 1992.

12. Gogate, S., Pant, R., and Arora, P.. "Incorporation of Some Cost and Economic Parameters in the Conceptual Design of an Air-Taxi Aircraft." AIAA Paper 94-4301. Sept. 1994.

13. Eason, E.. Nystrom, G., Burlingham. A.. and Nelson. E.. "Non-Hierarchic Multidisciplinary Design of a
Commercial Aircraft." AIAA Paper 944302, Sept. 1994.

14. Borland, C.. Kao, T.. Benton, J.. Mastro. R.. Frank. P.. and Barthelemy. J.-F.. "Muttidisciplinary Design Optimization of Commercial Aircraft Wing-An Exploratory Study." AIAA Paper 944305, Sept. 1994.

15. Grose, D., "Reengineering the Aircraft Design Process," AIAA Paper 94-4323. Sept. 1994.

16. Kroo, I., Altus, S.. Braun. R.. Gage. P., and Sobieski. I., "Multidisciplinary Optimization Methods for Aircraft Preliminary Design," AIAA Paper 94. 4325. Sept. 1994.

17. Brewer, J., Mavris, D., and Scrage. D.. "Implementation of Georgia Tech's Concurrent Engineering Methodology to a High-Speed Civil Transport (HSCT)." AIAA Paper 94-4329, Sept. 1994.

18. Huang. X., Dudley, J., Haftka. R.. Grossman. B., and Mason, W..

"Variable-Complexity Interfacing of Weight Equation and Structural Optimization for the Design of the HighSpeed Civil Transport." AIAA Paper 944.377, Sept. 1994.

19. Wakayama, S., and Kroo, I.. "Subsonic Wing Design Multidisciplinary Optimization," AIAA Paper 94-4409. Sept. 1994.

20. Lee, W., and Striz, A., "Multidisciplinary Optimization of a Transport Wing," AIAA Paper 94-4410. Sept. 1994.

21. Miller, G.. "An Active Flexible Wing Multi-Disciplinary Design Optimization Method," AIAA Paper 94-4412. Sept. 1994.

22. Holden. M., and Kroo, I., "A Collocation Method for Aeroelastic Optimization." AIAA Paper 96-3980, Sept. 1996.

23. Kolonay. R.. "Unsteady Aeroelastic Optimization in the Transonic Regime," AIAA Paper 96-3983, Sept. 1996.

24. Wakayama, S., Page. M., and Liebeck. R., "Multidisciplinary Optimization on an Advanced Composite Wing." AIAA Paper 96-4003, Sept. 1996.

25. Forster, E., Kolonay. R., and Venkayya, V., "Optimization of a Generic Fighter Wing Incorporating Active Aeroelastic Wing Technology," AIAA Paper 964010. Sept. 1996. 
26. Yan. S., and Striz. A., "Comparative Evaluation of Two MDO Codes in Aircraft Wing Analysis and Optimization," AIAA Paper 96-4032. Sept. 1996.

27. Chai. S., and Mason, W., "Landing Gear Integration in Aircraft Conceptual Design," AlAA Paper 96-4038. Sept. 1996.

28. Balabanov, V.. Kaufman, M.. Knill, D., Haim. D., Golovidov, O., Giunta, A.. Hattka, R. Mason. W., and Watson, L., "Dependence of Optimal Structural Weight on Aerodynamic Shape for High Speed Civil Transport," AIAA Paper 964046. Sept. 1996.

29. Young, J., Anderson, R., and Yurkovich, R.. "A Description of the F/A-18E/F Design and Design Process," AIAA Paper 98-4701. Sept. 1998.

30. Love, M., "Multidisciplinary Design Practices From the F-16 Agile Falcon," AIAA Paper 98-4704, Sept. 1998.

31. Bartholomew, P., "The Role of MDO Within Acrospace Design and Progress Towards and MDO Capability," AIAA Paper 98-4705. Sept. 1998.

32. Radovich, N.. and Layton. D.. "The F-22 Structural/Aeroelastic Design Process with MDO Examples," AIAA Paper 984732, Sept. 1998.

3. Hutchinson. M.. Lede. J.-C.. and Drela, M., "Multidisciplinary Design Optimization of the Theseus HighAltitude. Long-Endurance Unmanned Aircraft," AIAA Paper 98-4735. Sept. 1998.

34. Wakayama, S., and Kroo, I., "The Challenge and Promise of BlendedWing-Body Optimization," AIAA Paper 98-4736, Sept. 1998.

35. Giesing, J., and Barthelemy. J.-F., "A Summary of Industry MDO Applications and Needs". AIAA Paper 98-4737. Sept. 1998.

36. Chen, P., Sarhaddi. D., Liu, D.. Striz, A., and Jung, S., "ASTROS*: Seamless Integration of ASTROS with a Unified Aerodynamic Module," AIAA Paper 984739. Sept. 1998.

37. Rais-Rohani, M., and Greenwood, A.. "Product and Process Coupling in Multidisciplinary Design of Flight Vehicle Structures." AIAA Paper 98. 4820. Sept. 1998.
38. Eschenauer, H., Schumacher, A.. Honlinger, $H_{\text {., and Kiessling, F.. }}$ "Multidisciplinary Optimization Strategy-Tool for an Efficient Design Process of Large-Scale Aircraft." AIAA Paper 98-4823, Sepı. 1998.

39. Butler, R.. Hansson, E., Lillico, M.. and Van Dalen. F., "Comparison of MDO) Codes for Use in Conceptual and Preliminary Aircrafi Wing Design," AIAA Paper 98-4857. Sept. 1998.

40. Wood, R.. "A Discussion of Knowledge Based Technology for the Multidisciplinary Design of Military Aircraft," AlAA Paper 98-4944. Sep. 1998.

41. Walsh, J. L., Townsend, J. C., Salas, A., O., Samareh. J. A.. Mukhopadhyay, V., and Barthelemy. J.-F.. "Multidisciplinary High-Fidelity Analysis and Optimization of Aerospace Vehicles. Part 1: Formulation," AIAA Paper 2000-04l8. Jan. 2000.

42. Walsh. J. L., Weston, R. P.. Salas, A. O., Samareh, J. A., Mason. B. H., Green, L. L.. and Biedron, R. T.

"Multidisciplinary High-Fidelity Analysis and Optimization of Aerospace Vehicles, Part 2: Preliminary Results," AIAA Paper 2000-0419. Jan. 2000.

43. McCullers, A.. "Aircraft Configuration Optimization Including Optimized Flight Profiles." Proceedings of the Symposium on Recent Experiences in Multidisciplinar Analysis and Optimization, edited by J. Sobieski. NASA CP-2327. Hampton. VA. April 1984. pp. 396-412.

44. Allen. J., Vadde, S., and Mistree, F. "Modeling the Unknown in the Early Stages of Engineering Design." AIAA Paper 92-4755. Sept. 1992.

45. Khot, N., "Consideration of Robustness in Optimum Structural and Control Design." AIAA Paper 92-4762. Sept. 1992.

46. Pai. S., "Probabilistic Structural Analysis of Space Truss Structures for Nonuniform Thermal Environmental Effects." AIAA Paper 92-4769. Sept. 1992.

47. Frangopol, D.. and Iizuka, M.. "Probability-Based Structural System Design Using Multicriteria Optimization." AIAA Paper 92-4788, Sept. 1992. 
48. Teng, A.. Free. J., and Parkinson. A., "An Approach to Robust Design of Dynamic Systems-Allocation of Variation," AIAA Paper 92-4790, Sept. 1992.

49. Pritchard, J., Adelman. H., and Sobieski. J., "Optimization for Minimum Sensitivity to Uncertain Parameters," AIAA Paper 94-4279. Sept. 1994.

50. Ponslet, E.. Maglaras, G., Haftka. R.. Nikolaidis, E., Sensharma. P., and Cudney, H., "Analytical and Experimental Comparison of Probahilistic and Deterministic Optimization," AIAA Paper 94-4309. Sept. 1994.

51. Brown, S.. and Sepulveda. A.. "Approximation of System Reliabilities Using a Shooting Monte Carlo Approach." AIAA Paper 94-4310. Sept. 1994.

52. Santos, J., Siemasko. A.. Barros, P.. and Cardosa. J., "Interactive-Computer Aided Reliability Engineering," AIAA Paper 94-4312. Sept. 1994.

53. Su, J., and Renaud, J., "Automatic Differentiation in Robust Design." AIAA Paper 96-4005, Sept. 1996.

54. Su, J., and Renaud, J., "An Alternating Procedure for Efficient Reliability-Based Optimization," AIAA Paper 96-4007. Sept. 1996.

55. Lewis, K., and Mistree, F., "Modeling Interactions in Multidisciplinary Design: A Game Theoretic Approach." AIAA Paper 96-4060, Sept. 1996.

56. Liu, H., and Grandhi. R., "A ConvexTangent-Distance Algorithm and Second-Order Discriminant of the Most Probable Failure Point," AIAA Paper 964061, Sept. 1996.

57. Sues. R., Oakley, D., Rhodes, G.. and Hopkins. D., "MDO of Aeropropulsion Components Considering Uncertainty," AlAA Paper 96-4062. Sept. 1996.

58. Choi. K., Yu, X., and Chang. K.-H., “A Mixed Design Approach for Probabilistic Structural Durability." AIAA Paper 96-4063, Sept. 1996.

59. Chang, K.-H., Yu, X., and Choi, K. "Probabilistic Structural Durability Prediction," AIAA Paper 96-4064. Sept. 1996.

60. Chen, W.. Schrage, D., Allen, J., and Mistree. F.. "Statistical Experimentation for Affordable Concurrent Design," AIAA Paper 96-4085, Sept. 1996.

61. Marvis, D., Bandte, O.. and Schrage, D. "Application of Probabilistic Methods for the Determination of an Economically Robust HSCT Configuration." AIAA Paper 96-4090. Sept. 1996.

62. DeLaurentis, D.. Calise. A., Schrage. D. and Mavris. D., "Reduced Order Guidance Methods and Probahilistic Techniques in Addressing Mission Uncertainty," AIAA Paper 96-4174. Sept. 1996.

63. Gu, X., Renaud, J., and Batill, S., "An Investigation of Multidisciplinary Design Subject to Uncertainty." AlAA Paper 98-4747, Sept. 1998.

64. Braibant, V., Oudshoorn, A.. Boyer. C.. and Delcroix, F., "Non Deterministic "Possibilistic" Approaches for Structural Analysis and Optimal Design-A Comparison of Numerical Methods for Computing Structural Response Uncertainties," AIAA Paper 98-4750. Sept. 1998.

65. Cantelmi, F., Pelz, R., and Ogot. M.. "Stochastic Optimization for Preliminary Aircraft Design," AIAA Paper 98-4773. Sept. 1998.

66. Zink. P., Mavris, D.. Love, M., and Karpel, M.. "Robust Design for Aeroelastically Tailored/Active Aeroelastic Wing," AIAA Paper 984781, Sept. 1998.

67. Liaw, L., DeVries, R., and Cronin. D., "An MDO-Compatible Method for Robust Design of Vehicles, Systems, and Components." AIAA Paper 98-4786. Sept. 1998.

68. Liu. X.. Mahadevan, S., "System Reliability-Based Optimization of Composite Structures." AIAA Paper 984814. Sept. 1998.

69. DeVries, R.. "Development Needs for MDO, Robust Design and Target Tradeoff Analysis," AIAA Paper 984702, Sept. 1998.

70. Wu, J., "Development of a Fast Probability Analyzer for Large Numbers of Response Functions and Random Variables." AIAA Paper 98-4908, Sept. 1998.

71. Mangali. G.. Pieracci, A., and Rackwitz. R. "A Probablistic Approach for 
Integrated Structural Control Design," AIAA Paper 98-4911, Sept. 1998.

72. Kowal. M., and Mahadevan, S., "Uncertainty-Based Multidisciplinary Design Optimization." AIAA Paper 984915. Sept. 1998.

73. Chen, W., and Lewis, K., "A Robust Design Approach for Achieving Flexibility in Multidisciplinary Design," AIAA Paper 98-4945, Sept. 1998.

74. Thanedar. P. B., and Kodiyalam. S.. "Structural Optimization Using Probabilistic Constraints," Structural Optimization, Vol. 4, 1992, pp. 236-240 (also AIAA Paper 91-0922-CP, 1991).

75. Parkinson. A.. Sorensen, C., and Pourhassan, N.. “A General Approach for Robust Optimal Design." Joumal of Mechanical Design, Vol. 115, No. 1, 1993. pp. 74-80.

76. Chen, X., Hasselman, T. K., and Neill, D. J., "Reliability Based Structural Design Optimization for Practical Applications," AIAA Paper 97-1403. April 1997.

77. Du. X., and Chen. W.." Towards a Better Understanding of Modeling Feasibility Robustness in Engineering Design," American Society of Mechanical Engineers, Paper DAC-8565. Sept. 1999.

78. A Collection of Technical Papers, 40th AIAA/ASME/ASCE/AHS/ASC

Structures, Structural Dynamics, and Materials Conference and Exhibit. AIAA Forum on Non-Deterministic Approaches, St. Louis. MO, April $12-$ 15. 1999.

79. Alvin. K. F., Oberkampf, W. L.. Rutherford. B. M., and Diegart. K. V.. "Methodology for Characterizing Modeling and Discretization Uncertainties in Computational Simulation." SAND2000-0515, March 2000 .

80. Oberkampf, W. L.. DeLand, S. M., Rutherford. B. M.. Diegart, K. V.. and Alvin. K. F.. "Estimation of Total Uncertainty in Modeling and Simulation." SAND2000-0824. April 2000.

81. Forrest, B., Implementing Six Sigma. John Wiley \& Sons, New York 1999. pp 99-123.

82. Evans, M., Hastings, N., and Peacock. B. Statistical Distributions, 2nd Ed.. John Wiley \& Sons, New York, 1993.
83. McKay, M. D., "Evaluating Prediction Uncertainty," Los Alamos Report LA12915-MS, March 1995.

84. Trucano, T. G., "Prediction and Uncertainty in Computational Modeling of Complex Phenomena: A Whitepaper," SAND98-2776, Dec. 1998.

85. Robinson, D. G., "A Survey of Probabilistic Methods Used in Reliability, Risk and Uncertainty Analysis: Analytical Techniques I." SAND98-1189, June 1998.

86. Putko, M. M., Taylor, III, A. C.. Newman, P. A., and Green. L. L.. "Approach for Input Uncertainty Propagation and Robust Design in CFD Using Sensitivity Derivatives," Journal of Fluids Engineering, published by the Society of Mechanical Engineers. Vol. 124, No. 1, March 2002, pp. 60)-69 (see also.AIAA Paper 2001-2528. June 2001).

87. Huyse, L. and Lewis, M. R. "Aerodynamic Shape Optimization of Two-Dimensional Airfoils Under Uncertain Operating Conditions," ICASE Report No. 2001-1. NASA Langley Research Center Hampton, VA 23681-2199, Jan. 2001. pp. 13.

88. Huyse, L., "Free-Form Airfoil Shape Optimization Under Uncertainty Using Maximum Expected Value and SecondOrder Second Moment Strategies." ICASE Report No. 2001-18, NASA Langley Research Center Hampton. VA 23681-2199. January 2001, pp. 29.

89. Huyse, L.. and Walters, R. W., "Random Field Solutions Including Boundary Condtion Uncertainty for the SteadyState Generalized Burgers Equation," ICASE Report No. 2001-35. NASA Langley Research Center Hampton, VA 23681-2199. Jan. 2001, pp. 30.

90. Walters, R. W., and Huyse, L., "Uncertainty Analysis for Fluid Mechanics with Applications". NASA/CR-2002-21।449. ICASE Report No. 2002-1. NASA Langley Research Center Hampton, VA 23681-2199, Jan 2001. pp. 50 .

91. Gumbert, C. R.. Newman, P. A., and Hou, G. J.-W.. "Effect of Random Uncertainty on the Computational Design of a 3-D Flexible Wing," AIAA Paper 2002-2806. June 2002. 
92. Chinchalkar, S., and Taylor, D. L.. "Geometric Uncertainties in Finite Element Analysis." Computing Systems in Engincering. Vol. 5, No. 2, 1994, pp. 159- 170 .

93. Du, X., and Chen.W. "Methodology for Managing the Effect of Uncertainty in Simulation-Based Design." AIAA Joumal, Vol. 38. No. 8. 2000, pp. 1471-1478.

94. Madsen. O.. Krenk, S.. and Lind, N.C.. Methods of Structural Safety, PrenticeHall. Inc. Englewood Cliffs. N.J., 1986

95. Der Kiureghian. A., Lin, H.-Z., and Hwang. S.-J., "Second-Order Reliability Approximations," Journal of Engineering Mechanics, Vol. 113, No. 8, 1987. pp. 1208-1225.

96. Lin, H.-Z., and Khalessi. M. R.. "Identification of the Most-ProbablePoint in Original Space-Applications to Structural Reliability." Proceedings of the 34th Structures, Structural Dyamics, and Materials Conference. AIAA/ASME/ASCE/AHS/ASC, La Jolla, California, April 19-22, 1993, pp. 2791-2800

97. Khalessi, M.R., Lin. H.-Z., and Trent. D. J., "Development of the FEBREL Finite Element-Based Reliability Computer Program," Proceedings of the 34th Structures, Structural Dymamics, and Materials Conference,

AIAA/ASME/ASCE/AHS/ASC, La Jolla. California, April 19-22, 1993, pp. $753-761$.

98. Torng, T.Y.. Lin. H.-Z., Chang, C., Khalessi, M.R., and D'Angelo, A.. "Development of the Integrated Probabilistic Analysis Software Package. FEBREL-MSC/NASTRAN." Proceedings of the $36 \mathrm{th}$ Structures. Structural Dinamics, and Materials Conference. AIAA/ASME/ASCE/AHS/ASC, New Orleans, LA April 10-13, 1995.

99. Lin. H.-Z. and Khalessi, M. R., "Calculation of Failure Probability by Using X-Space Most-Probable-Point," Proceedings of the 34 th Structures, Structural Dinamics, and Materials Conference, AIAA / ASME / ASCE / AHS / ASC, La Jolla, California, April 19-22. 1993, pp. 2801-2808.

100. Bischof, C., Carle, A., Corliss, G., Griewank, A., and Hovland, P.,
"ADIFOR - Generaling Derivative Codes from Fortran Programs." Scientific Programming. No. 1, 1992, pp. $1-29$.

101. Bischof, C.. and Gricwank. A.. "ADIFOR: A Fortran System for Portable Automatic Differentiation," AIAA Paper 92-4744. Sept. 1992.

102. Bischof, C.. Knauff Jr., T., Green, L., and Haigler, K., "Parallel Calculation of Sensitivity Derivatives for Aircraft Design Using Automatic Differentiation," AIAA Paper 94-4261. Sept. 1994.

103. Bischof, C., Carle, A., Khademi, P.. and Mauer. A., "Automatic Differentiation of FORTRAN," Computational Science \& Engineering, Vol. 3. No. 3, Fall 1996. pp. 18-32.

104. Carle, A.. Fagan, M., and Green, L.. -Preliminary Results from the Application of Automatic Adjoint Code Generation to CFL3D," AIAA Paper 984807, Sept. 1998

105. Carle, A.. and Fagan. M.. "Overview of Adifor 3.0." Department of Computational and Applied Mathematics, Rice University, CAAMTR 00-02. Jan. 2000)

106. Marsgalia. G., and Zaman, A., "Some Portable Very-Long Period Random Number Generators." Computers in Phrsics, Vol. 8. No. 1. 1994. pp. 117121.

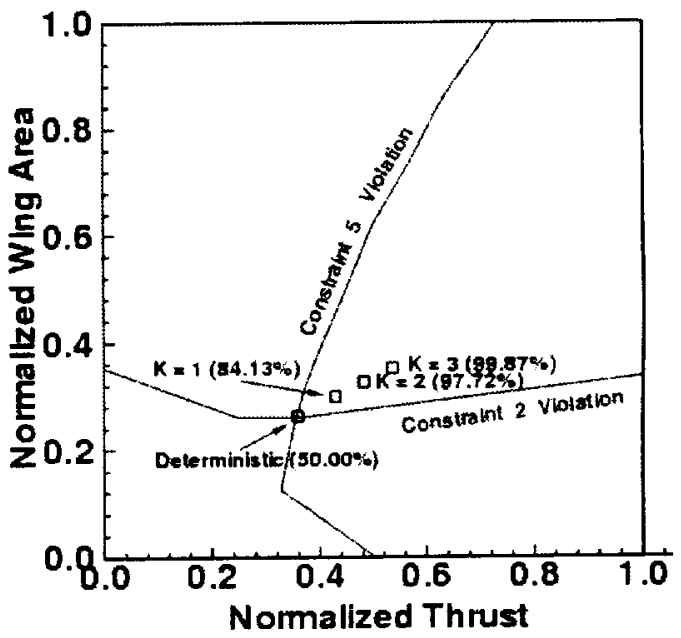

Fig. 1. Design points and active constraint boundaries for deterministic and robust optimization, c.o.v. $=5 \%$. 


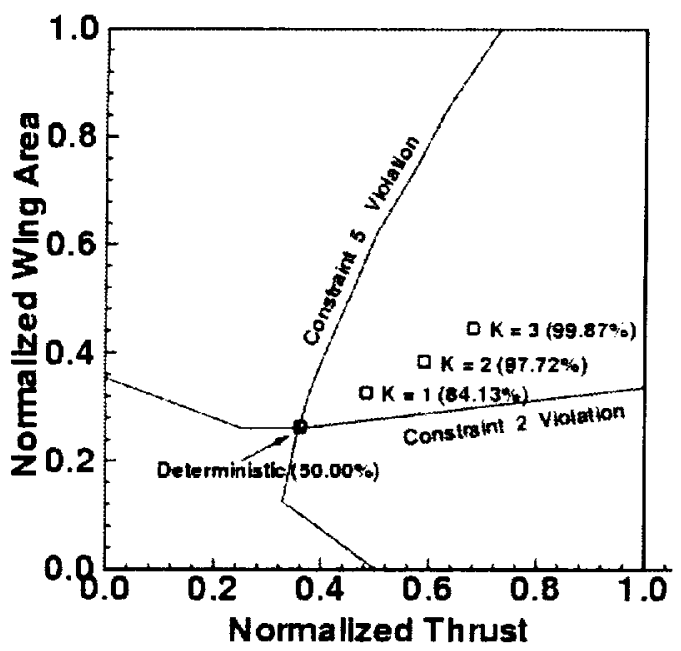

Fig. 2. Design points and active constraint boundaries for deterministic and robust optimization. c.o.v. $=10 \%$.

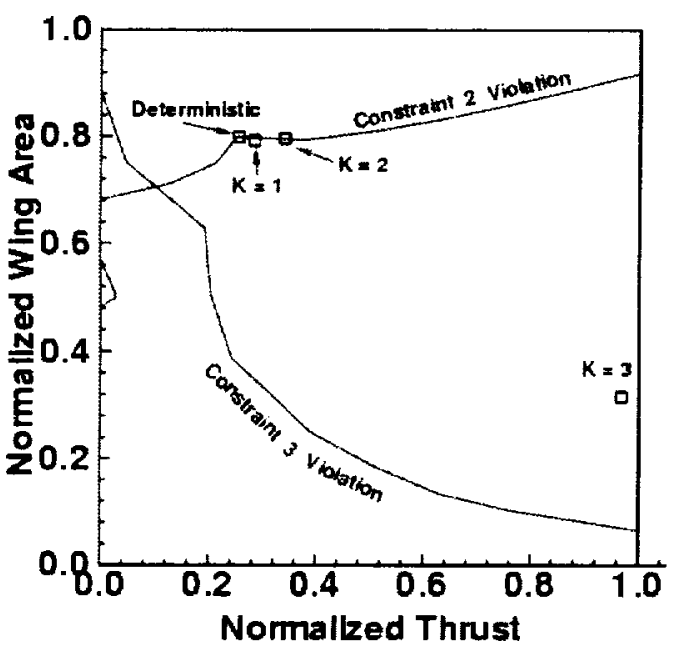

Fig. 3. Design points and active constraint boundaries for deterministic and robust optimization. c.o.v. $=.05 \%$.

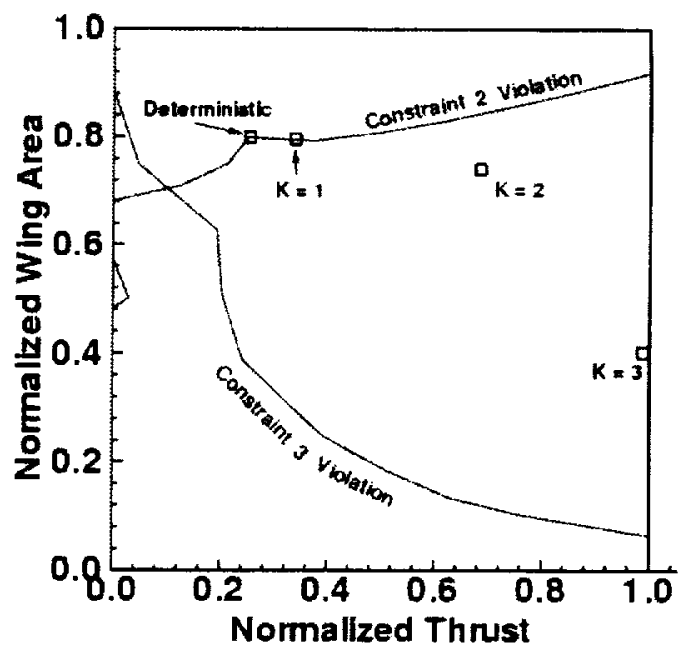

Fig. 4. Design points and active constraint boundaries for deterministic and robust optimization, c.o.v. $=.08 \%$.

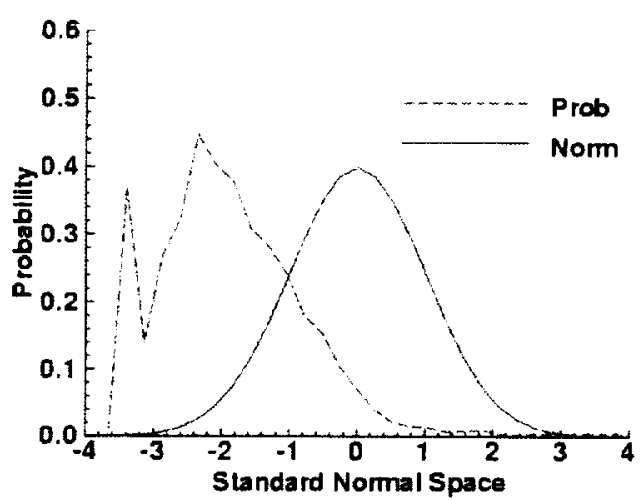

Fig. 5. Aircralt gross weight distribution plot. supersonic. two variable, deterministic design case. 


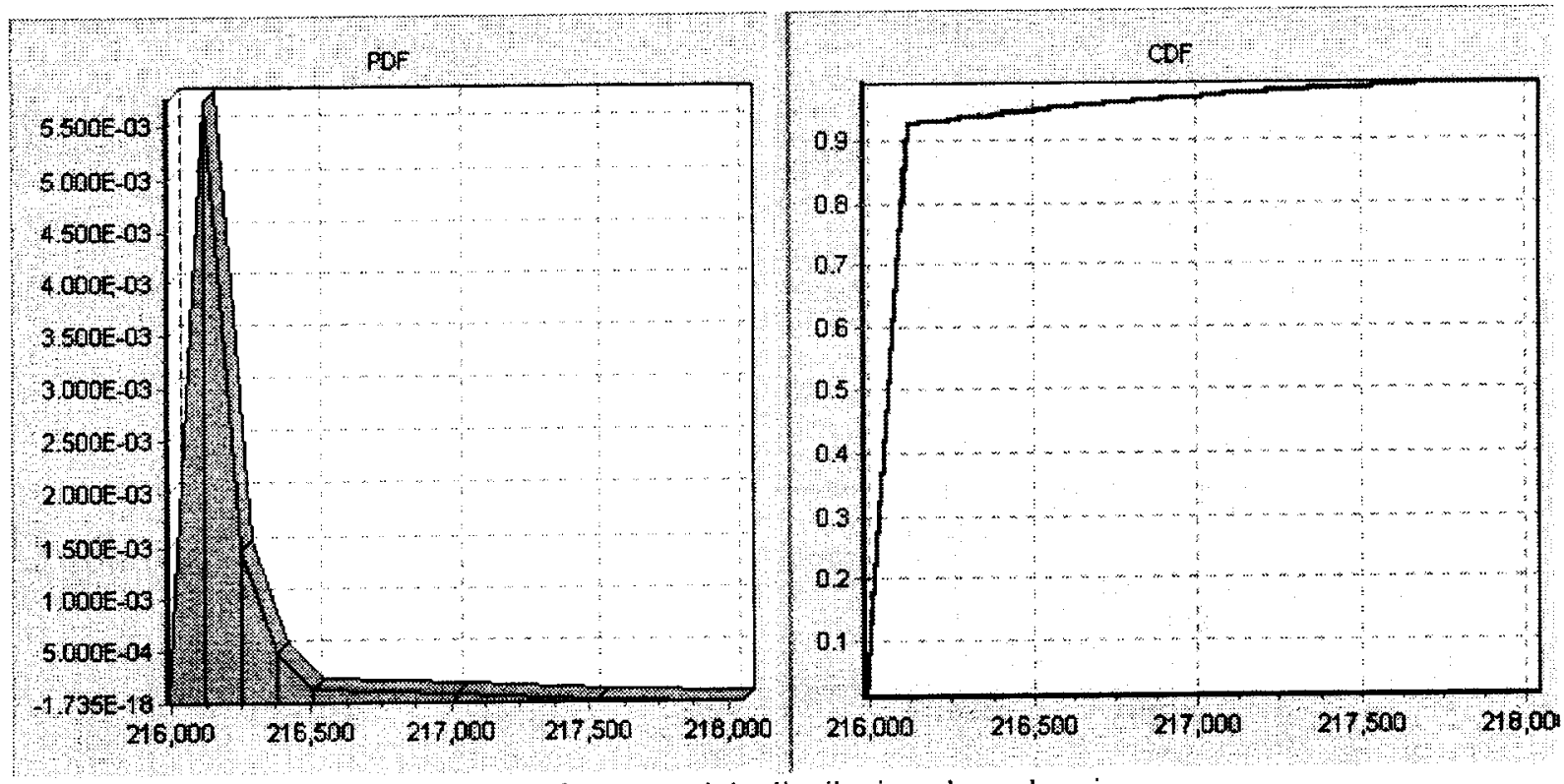

Fig. 6. Aircraft gross weight distribution plot, subsonic, two variable, deterministic design case. c.o.v. $=5 \%$.
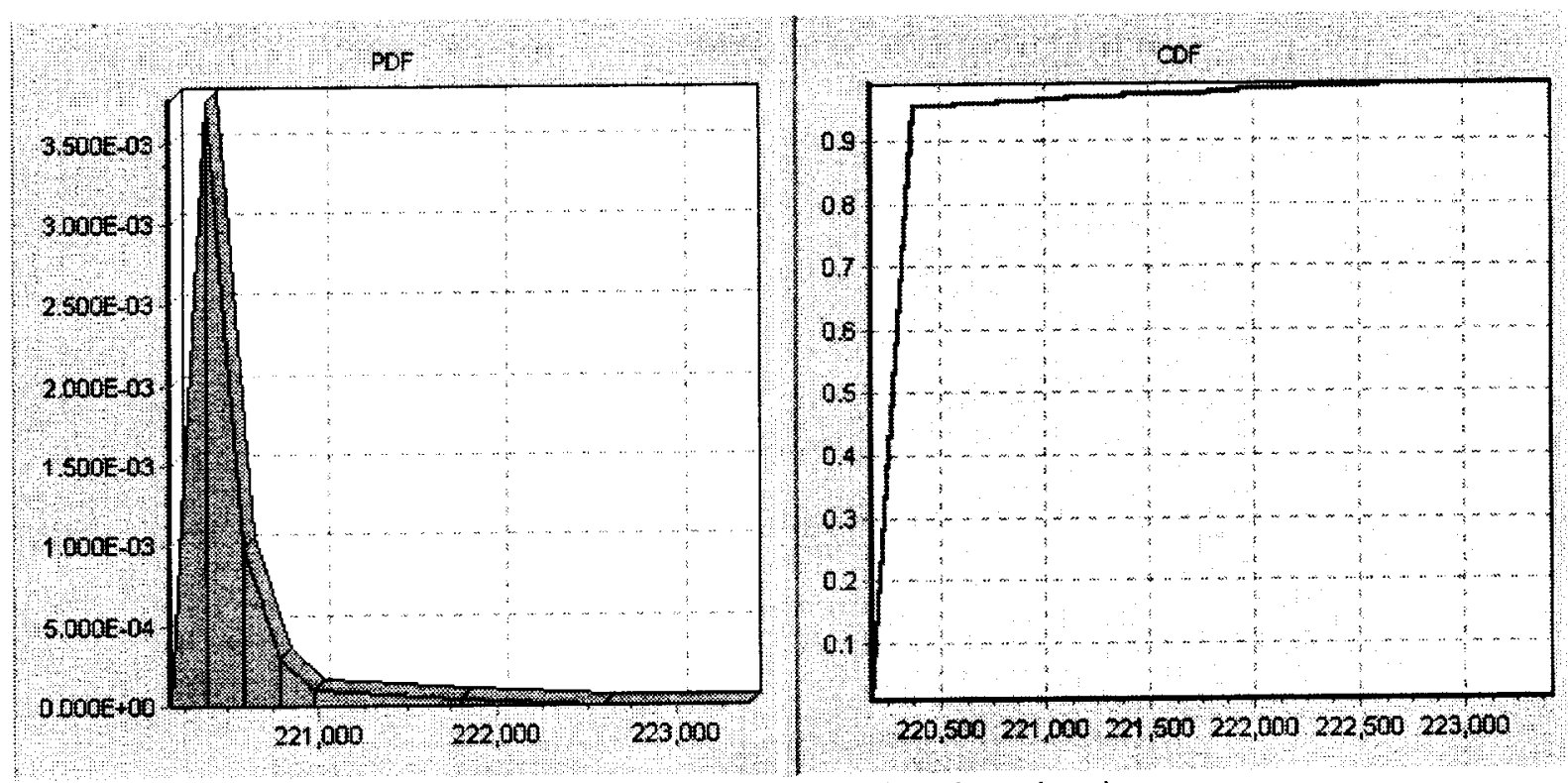

Fig. 7. Aircraft gross weight distribution plot, subsonic. two variable. deterministic design case, c.o.v. $=10 \%$. 


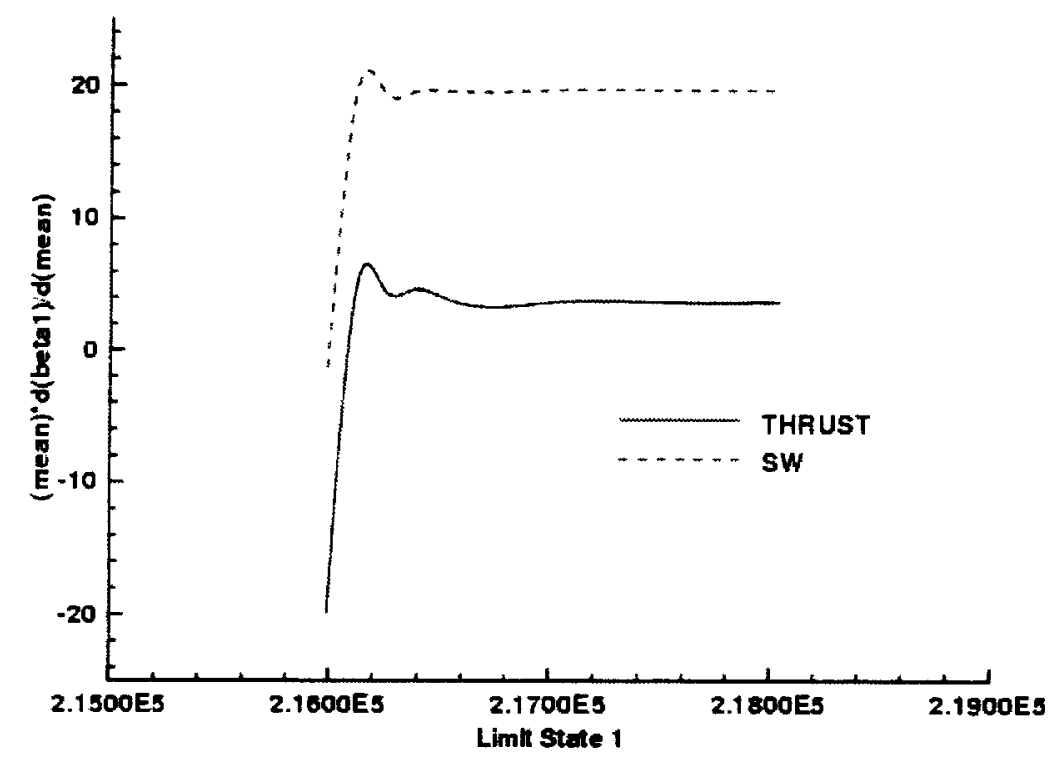

Fig. 8. Reliability index sensitivities with respect to random input variable mean values. scaled by the random input variable mean value. c.o.v $=5 \%$.

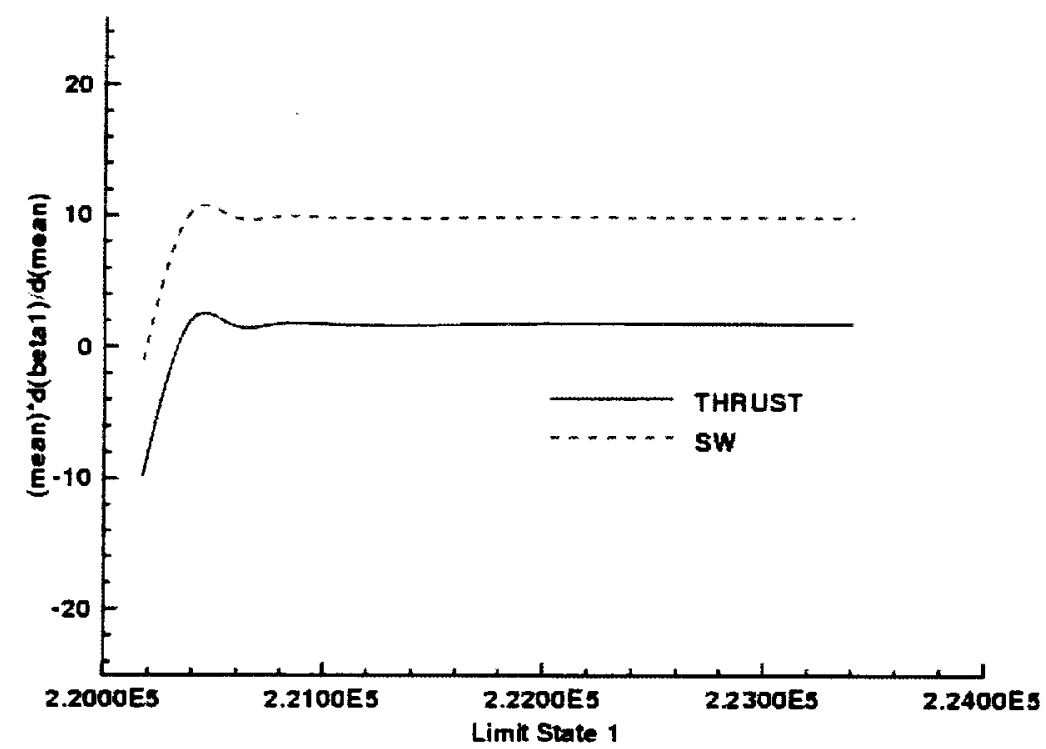

Fig. 9. Reliability index sensitivities with respect to random input variable mean values, sealed by the random input variable mean value, c.o.v $=10 \%$. 
